\title{
Gravity with a dynamical preferred frame
}

\author{
Ted Jacobson* and David Mattingly ${ }^{\dagger}$ \\ Department of Physics, University of Maryland, College Park, MD 20742-4111, USA
}

We study a generally covariant model in which local Lorentz invariance is broken by a dynamical unit timelike vector field $u^{a}$ - the "aether". Such a model makes it possible to study the gravitational and cosmological consequences of preferred frame effects, such as "variable speed of light" or high frequency dispersion, while preserving a generally covariant metric theory of gravity. In this paper we restrict attention to an action for an effective theory of the aether which involves only the antisymmetrized derivative $\nabla_{[a} u_{b]}$. Without matter this theory is equivalent to a sector of the EinsteinMaxwell-charged dust system. The aether has two massless transverse excitations, and the solutions of the model include all vacuum solutions of general relativity (as well as other solutions). However, the aether generally develops gradient singularities which signal a breakdown of this effective theory. Including the symmetrized derivative in the action for the aether field may cure this problem.

\section{INTRODUCTION}

The Lorentz group is non-compact, since the boost parameter is unbounded. This makes exact Lorentz invariance impossible to test uniformly. Lorentz invariance has thus been tested only up to some maximum boost and beyond that lies an infinite volume of uncharted territory in the Lorentz group. Contrast this with the rotation group. Rotation invariance can be tested by filling in the compact $S O(3)$ group manifold more and more densely with data points, or by checking a few randomly selected rotations. The rotation group can be and has been uniformly explored.

There is also reason to doubt exact Lorentz invariance: it leads to divergences in quantum field theory associated with states of arbitrarily high energy and momentum. This problem can be cured with a short distance cutoff which, however, breaks Lorentz invariance.

For these reasons we entertain the possibility that there is a preferred rest frame at each spacetime point. In particular, we seek a viable effective field theory incorporating a breaking of local Lorentz invariance.

If the preferred frame were to be a fixed external structure, then it would violate general covariance, which would require us to abandon general relativity (or any

*jacobson@physics.umd.edu

†davemm@physics.umd.edu generally covariant modification thereof). General covariance ordinarily implies that the divergence of the matter energy-momentum tensor $T_{a b}$ vanishes when the matter field satisfies its equation of motion. This is required for consistency of the Einstein field equation $G_{a b}=8 \pi G T_{a b}$, since the divergence of the Einstein tensor $G_{a b}$ is identically zero by virtue of the contracted Bianchi identity. If a fixed preferred frame is introduced into the matter action, for example, general covariance is lost, $T_{a b}$ is not divergenceless, and the Einstein equation is inconsistent. We therefore seek to incorporate the preferred frame while preserving general covariance, which requires that the preferred frame be dynamical.

It would be most appealing if the preferred frame were somehow determined by the spacetime metric itself. As discussed below, in spacetimes with an initial singularity the metric can be used to define a cosmological time function, the gradient of which then determines a preferred frame (which is by construction timelike). However, the nonlocal relationship between this frame and the metric results in infinitely nonlocal field equations if this frame is incorporated into the action principle. Theories with even a finite amount of nonlocality are generally horribly unstable [1], so we do not consider this a viable approach.

To avoid such unacceptable behavior the preferred frame should arise from local conditions, which of course reflect conditions at earlier times but only through $d y$ namics. For example, this dynamical frame could be defined by a vector field [3, 2, 4, 5] or by the gradient of a scalar field [6,7]. In these approaches the presence or absence of a preferred frame depends on the field configuration, since the preferred vector may vanish or may not be timelike.

Since our motivation arises from doubts about the fundamental validity of exact Lorentz invariance, we are not interested in a theory possessing a Lorentz invariant phase. We wish to study instead an effective theory in which there is always a preferred frame. This frame is defined by a timelike direction or, equivalently, by a unit timelike (contravariant or covariant) vector field.2 Such a field carries a nonlinear representation of the local Lorentz group since the field takes values not in a vector space but on the unit hyperboloid in the tangent

\footnotetext{
${ }^{1}$ We use units with $c=1$ and the metric signature $(+---)$.

${ }^{2} \mathrm{~A}$ unit timelike vector contains a discrete piece of information that a frame by itself does not have, namely, a time orientation. The theory may or may not depend on this orientation.
} 
space. This could therefore be called a theory of nonlinearly realized Lorentz invariance. It is analogous to a gauge theory with a non-linear sigma model Higgs field of fixed norm [8,9]. There seems to be no generally accepted terminology for this sort of symmetry structure. Since the symmetry breaking unit vector field is not a state-dependent expectation value but rather breaks this symmetry in all states, it may be misleading to say the symmetry is "spontaneously broken". For lack of a better idea we shall take refuge in ambiguity and just call it "broken". What this really means is that in order to implement the local Lorentz symmetry one must transform not only the matter fields but also the background unit vector, so for practical purposes it will appear as what would normally be called broken Lorentz symmetry.

The theory described here was devised about a decade ago by J.C. Dell together with one of us [10], and we have since learned that similar ideas have independently been studied previously. The non-gravitational part of the theory (and generalizations thereof) was considered by Dirac [11] in the early 1950's as a new theory of electrons (in which the unit timelike vector played the dual role of gauge-fixed vector potential and flow vector of a stream of charged dust). A class of generally covariant theories breaking Lorentz invariance was studied by Gasperini 12] in many papers. In this work the tetrad formalism was used, and the local Lorentz symmetry was broken by including in the action terms referring to a fixed "internal" unit timelike vector. This is equivalent to our formulation in terms of the metric and a unit timelike vector. To see the equivalence, note that the tetrad defines a metric and associates to the fixed internal vector a unit vector field on spacetime. The only other information in the tetrad is the gauge freedom parametrized by the local rotations leaving invariant the preferred timelike vector. Eliminating this gauge freedom leads to the formalism used in this paper. Gasperini has studied both cosmological and central field solutions, with various choices for the specific form of the second derivative terms in the Lagrangian. In the present paper we focus on a different Lagrangian.

The particular Lagrangian studied here was also considered by Kostelecký and Samuel [13], as a simplified model of the spontaneous Lorentz symmetry breaking that might occur in string theory, although in Ref. 13 the preferred vector was not necessarily timelike. More generally, those authors argued that spontaneous Lorentz symmetry breaking in string theory may produce vacuum expectation values of more than one tensor field. In this case, rather than having just a single "preferred frame" there might be several background Lorentz tensors which collectively break part or all of the Lorentz symmetry.

The remainder of this paper is organized as follows. In section II we explain the nonlocality problem encountered if a cosmological time is used to define the preferred frame. In section III our proposed field theory of a preferred frame is formulated and its general properties are investigated. It is seen that the solutions for our the- ory comprise a subset of the solutions to the coupled Einstein-Maxwell-charged dust equations. Several types of exact solutions to the field equations are characterized in section IV, and the linearized theory is studied in section $\mathrm{V}$. Coupling of the preferred frame to matter fields is discussed in section VI. Both the dimension $\leq 4$ couplings and some higher dimension ones are examined. The paper concludes with a brief discussion in section VII.

Among the dimension $>4$ couplings are included theories involving Lorentz non-invariant dispersion at high wavevectors. This is motivated by recent work in which high frequency dispersion is invoked to avoid the role of trans-Planckian modes in the Hawking effect (for a review see [14]). In this framework such theories can be formulated in a generally covariant manner so that gravitational effects can be consistently incorporated. Higher dimension couplings also also provide an alternative generally covariant formulation of variable speed of light models, in which different fields propagate at different speeds possibly at different cosmological epochs. Such models have recently been of interest as potential alternatives to standard cosmology, and have been given generally covariant formulations using additional fields to define the preferred frame [5].

\section{COSMOLOGICAL TIMES}

In this section we briefly describe the construction of a cosmological time function determined purely by the metric, and the reason for rejecting it for the purposes of an effective theory of local Lorentz symmetry breaking.

The cosmological metric of our universe, by virtue of its (approximate) homogeneity, (approximately) defines a preferred spacelike foliation of spacetime. However, this particular definition of the time function relies on the symmetry of the spacetime. For a workable theory with general covariance what is needed is a definition of cosmological time that can be used independently of symmetry.

It is difficult to think of a notion of cosmological time that would make sense for all possible cosmologies. However, if we restrict attention to spacetimes with a "beginning", then two notions of cosmological time at $x$ present themselves: $(i)$ volume time, the spacetime volume (or perhaps the fourth root thereof) of the past set $I^{-}[x]$, and (ii) maximal time, the maximal proper time along a causal curve going back to the initial singularity. Other

\footnotetext{
${ }^{3}$ The maximal time function has been discussed in Refs. [15 17]. In particular, a powerful theorem proved in Ref. 117. establishes a number of properties of this function under the further assumption that the initial singularity is the only place past directed causal curves can end.
} 
possibilities are combinations or smoothed averages of these times. Both of these time functions are determined non-locally but causally by the spacetime to the past of $x$. They may or may not be sufficiently smooth functions to enter meaningfully into a local action principle. If If we assume that they are indeed sufficiently smooth we find that there is in any case a fatal problem with using them in this manner, as will now be explained.

Suppose that to the usual action for gravity and matter fields is added a term involving one of the above cosmological times,

$$
S=S_{\text {local }}+S_{\tau}
$$

We assume that the equations of motion are obtained as usual by requiring that the action is stationary with respect to variations of the fields. The variational derivative of the action with respect to $g_{a b}(x)$ has the form

$$
\frac{\delta S}{\delta g_{a b}(x)}=\frac{\delta S_{\text {local }}}{\delta g_{a b}(x)}+\frac{\delta^{\prime} S_{\tau}}{\delta g_{a b}(x)}+\int d^{4} x^{\prime} \frac{\delta S_{\tau}}{\delta \tau\left(x^{\prime}\right)} \frac{\delta \tau\left(x^{\prime}\right)}{\delta g_{a b}(x)},
$$

where the prime on $\delta^{\prime}$ indicates that the metric dependence of $\tau(x)$ is not included in the variation. Since $\delta \tau\left(x^{\prime}\right) / \delta g_{a b}(x)$ has support when $x^{\prime}$ lies to the future of $x$, the field equation $\delta S / \delta g_{a b}(x)=0$ involves the values of the fields to the future of $x$. Indeed the metric field equation is infinitely non-local in time, since the time function at any point to the future can be affected by a metric variation at $x$. Even finite nonlocality in time leads to unphysical instability [1], so this approach to incorporating a preferred frame must be rejected. If the action depends on $\tau$ only through its derivative $\nabla_{a} \tau$, then the equation of motion would be causal if $\delta \nabla_{c} \tau\left(x^{\prime}\right)$ depended only on $g_{a b}(x)$ at $x=x^{\prime}$. However, this is not the case for the either the volume time or the maximal time.

\section{AETHER DYNAMICS}

We now turn to a class of theories in which there is a preferred frame which is determined by a local field. It is convenient to give a name to this field, and "aether" seems as good a name as any. Let us take the aether field to be a unit timelike vector field $u^{a}$, which is dimensionless, like the metric. To handle the condition that $u^{a}$ is a unit vector, we include in the action a Lagrange multiplier term. Note that we are implicitly assuming that the

\footnotetext{
${ }^{4}$ One of the results of the theorem of Ref. 17] referred to in the previous footnote is that the maximal time function is locally Lipschitz and its first and second derivatives exist almost everywhere. The volume time function may well be even better behaved.
}

spacetime admits a globally defined unit timelike vector field which is the case if and only if the spacetime is time orientable.

\section{A. Action}

The most general Lagrangian involving the metric and the aether with two or fewer derivatives is, up to a total divergence,

$$
\begin{aligned}
\mathcal{L}_{g, u}= & a_{0}-a_{1} R-a_{2} R_{a b} u^{a} u^{b} \\
& -b_{1} F^{a b} F_{a b}-b_{2}\left(\nabla_{a} u_{b}\right)\left(\nabla^{a} u^{b}\right)-b_{3} \dot{u}^{a} \dot{u}_{a},
\end{aligned}
$$

where $\dot{u}^{a}:=u^{m} \nabla_{m} u^{a}$, and $F_{a b}$ is defined in analogy to the electromagnetic field strength,

$$
F_{a b}:=2 \nabla_{[a} u_{b]} .
$$

The term $\left(\nabla_{a} u^{a}\right)^{2}$ is equivalent, via integration by parts, to the combination $\left(\nabla_{a} u_{b}\right)\left(\nabla^{a} u^{b}\right)-(1 / 2) F^{a b} F_{a b}+$ $R_{a b} u^{a} u^{b}$, so has not been included in (3.1).

The Lagrangian (3.1) is similar to the one discussed in Ref. 18] as the most general Lagrangian for a vectortensor theory of gravity including terms up to second order in derivatives and quadratic in the vector field. The differences are that (i) the terms $u^{2}$ and $R u^{2}$ are missing from our action since the vector field is constrained to be a unit vector, and (ii) we have included the quartic term $b_{3} \dot{u}^{2}$, which was omitted in Ref. [18] because it is not quadratic in $u^{a}$. 19] Note that, even without the last term, our theory is not a special case of the vector-tensor theories discussed in Ref. [18], since the constraint $u^{2}=1$ affects the field equations.

The coefficient $a_{0}$ in the action (3.1) has mass dimension 4 while $a_{1,2}$ and $b_{1,2,3}$ have mass dimension 2 . Lacking the underlying fundamental theory we do not try to assign a priori the values of these coefficients. A partial analysis of the observational consequences and limits on them has been done for the vector-tensor theories [18], however that analysis does not apply directly to our case due to the presence of the constraint term. It is fairly clear nevertheless that whatever values $b_{1,2,3}$ take, agreement with observation will require that $a_{2} \ll a_{1}$, and that $a_{0} / a_{1}$ (which is basically the cosmological constant) must not be much larger than the squared Hubble constant.

In this initial foray we shall restrict attention to the simple case in which the only terms with non-zero coefficients are $R$ and $F^{2}$. That is, we set $a_{0}=a_{2}=b_{2}=$ $b_{3}=0$. The minimal theory we consider is thus defined by the action

$$
\begin{aligned}
& S_{\text {min }}\left[g_{a b}, u^{a}, \lambda\right]=\int d^{4} x \sqrt{-g} \\
& \left(-a_{1} R-b_{1} F^{a b} F_{a b}+\lambda\left(g_{a b} u^{a} u^{b}-1\right)\right) .
\end{aligned}
$$


This minimal theory is one of the models considered by Kostelecký and Samuel 13] in the paper mentioned in section I. Those authors studied a broader class of models in which $u^{a}$ is not necessarily constrained to be a unit vector but rather possesses a Lorentz-invariant potential energy with a minimum at some fixed value of $u_{a} u^{a}$. They also allowed for extra, compact spatial dimensions of spacetime, and examined cases where the symmetry breaking vector lies in the extra dimensions as well as cases where it lies in the four ordinary spacetime dimensions. Our paper by contrast is restricted to four dimensions and to a timelike vector of fixed norm. Later in this paper we shall also add matter terms to the action, including terms which couple the aether field to the matter.

Note that $F_{a b}$ is invariant under the "gauge transformation"

$$
u_{a} \rightarrow u_{a}+\nabla_{a} f,
$$

however the constraint $u^{2}=1$ does not share this symmetry (nor do the additional couplings in general), so the theory is certainly not "gauge invariant". The constraint does have a limited version of this symmetry however, namely for those functions $f$ satisfying $\left(u_{a}+\nabla_{a} f\right)\left(u^{a}+\nabla^{a} f\right)=u_{a} u^{a}=1$. The general solution to this equation is

$$
u^{a} \nabla_{a} f=-1 \pm \sqrt{1+q^{a b} \nabla_{a} f \nabla_{b} f}
$$

where

$$
q_{a b}:=-g_{a b}+u_{a} u_{b}
$$

is the (positive definite) spatial metric orthogonal to $u^{a}$. Thus the action (3.3) is invariant under the gauge transformation (3.4) if $f$ is chosen arbitrarily on a spacelike surface and then determined uniquely elsewhere (up to a discrete choice of sign) by integration of (3.5) along the flow of $u^{a}$.

\section{B. Field equations}

The equations of motion arising from the action (3.3) are

$$
\begin{gathered}
G_{a b}=-\frac{2 b_{1}}{a_{1}}\left(F_{a m} F_{b}{ }^{m}-\frac{1}{4} F^{2} g_{a b}\right)+\frac{\lambda}{a_{1}} u_{a} u_{b}, \\
\nabla_{a} F^{a b}=-\frac{\lambda}{2 b_{1}} u^{b}, \\
g_{a b} u^{a} u^{b}=1 .
\end{gathered}
$$

The metric equation (3.7) has the form of the Einstein equation $G_{a b}=8 \pi G T_{a b}$, where $G=1 / 16 \pi a_{1}$, and the stress tensor receives contributions from both the $F^{2}$ term and the constraint term in the action. (The constraint equation (3.9) has been used to drop the contribution to (3.7) that would have come from the variation of $\sqrt{-g}$ in the constraint term.) The contribution from the constraint term looks like that of a (pressureless) dust with rest energy density $2 \lambda$, and that from the $F^{2}$ term is the usual Maxwell tensor familiar from electromagnetism, if we identify the vector potential as

$$
\text { " } A_{m} " \leftrightarrow 2 \sqrt{b_{1}} u_{m} .
$$

The stress tensor thus satisfies the usual energy conditions provided $b_{1} / a_{1}$ and $\lambda / a_{1}$ are positive.

In terms of the vector potential $A_{m}$ (3.10) the constraint equation (3.9) becomes

$$
A_{m} A^{m}=4 b_{1}
$$

which can be interpreted as a gauge condition. The aether field equation (3.8) becomes the Maxwell equation with source equal to the current of a charged dust fluid with 4 -velocity $u_{b}$ and charge density $-\left(\lambda / \sqrt{b_{1}}\right)$. The evolution of $\lambda$ is determined by the current conservation equation which follows from divergence of the aether field equation (3.8) upon using the identity $\nabla_{a} \nabla_{b} F^{a b} \equiv 0$. Thus $\lambda$ satisfies a first order ordinary differential equation along the flow lines of $u^{a}$ :

$$
u^{a} \nabla_{a} \lambda=-\lambda \nabla_{a} u^{a} .
$$

In particular, if $\lambda$ vanishes on a Cauchy surface, it must vanish everywhere. Also, the sign of $\lambda$ on a given flow line cannot change, since if $\lambda=0$ at any point on a flow line it must vanish everywhere on that line.

\section{Relation to Einstein-Maxwell-charged dust system}

We have just seen that the field equations of the minimal theory take the form of the coupled Einstein-Maxwell equations, with a charged dust matter source possessing charge to mass ratio $-1 / 2 \sqrt{b_{1}}$. There is no explicit equation of motion for the dust, however the normalization condition (3.9) provides such an equation. Taking the gradient of $u^{2}=1$ we have

$$
\begin{aligned}
0 & =\nabla_{a}\left(u^{b} u_{b}\right) \\
& =2 u^{b} \nabla_{a} u_{b} \\
& =2\left(u^{b} \nabla_{b} u_{a}+u^{b} F_{a b}\right) .
\end{aligned}
$$

Let us define $\tilde{F}_{a b}=2 \partial_{[a} A_{b]}=2 \sqrt{b_{1}} F_{a b}$. Then (3.15) becomes

$$
u^{b} \nabla_{b} u_{a}=-\frac{1}{2 \sqrt{b_{1}}} \tilde{F}_{a b} u^{b},
$$

which is the equation of motion for a particle in the electromagnetic field $\tilde{F}_{a b}$, with charge to mass ratio $-1 / 2 \sqrt{b_{1}}$, the same ratio we inferred from the Einstein 
and Maxwell equations! Thus any solution of our minimal theory is a solution of the Einstein-Maxwell-charged dust equations (although the converse is not true). The equivalence to a subset of the charged dust solutions demonstrates that the equations of our theory admit an initial value formulation, and it provides some useful intuition about the nature of the solutions.

Our theory is not equivalent to the Einstein-Maxwellcharged dust system because in the general solution of that system the dust 4-velocity is not proportional to the vector potential in some gauge. That is, although there is always a gauge transformation that will make $A_{m} / 2 \sqrt{b_{1}}$ a unit vector, it cannot in general be made to coincide with the dust 4 -velocity.

\section{SOLUTIONS}

In this section we characterize a few types of solutions to the field equations.

\section{A. Solutions with $\lambda=0$}

If $\lambda=0$, then the two field equations (3.7, 3.8) are just the Einstein-Maxwell equations. Any solution to these equations is a solution in our theory provided a gauge can be chosen so that the constraint equation (3.11) is satisfied. Such a gauge always exists, at least locally.

\section{B. Solutions with $F_{a b}=0$}

A special class of solutions to the field equations with $\lambda=0$ are those with $F_{a b}=0$. For such fields, (3.8) implies that $\lambda=0$, and the field equations (3.7.3.9) reduce to the ordinary vacuum Einstein equation together with the constraint $u^{2}=1$. When $F_{a b}=0$ it follows, at least locally, that $u_{a}=\nabla_{a} \tau$ for some function $\tau$, and the constraint then implies that $\nabla_{a} \tau \nabla^{a} \tau=1$. The general solution for such a function $\tau$ can specified by assigning the value $\tau=0$ to an arbitrary spacelike surface, and determining $\tau$ elsewhere by "uniform normal extension", i.e. by the differential equation $n^{a} \nabla_{a} \tau=1$, where $n^{a}$ is the unit normal to the surface.

\footnotetext{
${ }^{5}$ The general form of the discrepancy between these two 4vectors was found by Dirac (see the second paper of Ref. 11]), who showed that (in four spacetime dimensions) there is always a gauge in which $A_{m} / 2 \sqrt{b_{1}}=u_{m}+\xi \nabla_{m} \eta$, where $\xi$ and $\eta$ are functions that are constant along the flow lines of $u^{a}$. Dirac included the functions $\xi$ and $\eta$ as dynamical variables in order to obtain a theory in which arbitrary electron streams were admitted. In the third paper of Ref. 11] he allowed for multiple streams.
}

Another way to think of this construction is in terms of the congruence of integral curves of $u^{a}$. When $F_{a b}=0$, eqn. (3.15) implies that these curves are geodesics. Moreover, if $u^{a}$ is the unit tangent field to a congruence of geodesics, then $F_{a b}=2 \nabla_{[a} u_{b]}=0$ if and only if the congruence is hypersurface-orthogonal. Hence the general solution of this type is just an arbitrary solution to the Einstein equation, together with $u^{a}$ given by the unit tangent field of any hypersurface-orthogonal congruence of timelike geodesics in this metric. A special case is flat spacetime, where the $u^{a}$ congruence consists of straight lines normal to an arbitrary initial spacelike hypersurface.

\section{Singular aether evolution}

This characterization of the $F_{a b}=0$ solutions shows that, at least for such solutions, the evolution of $u_{a}$ is generally singular. The geodesics launched normally from a spacelike surface will typically cross. Where they do, the quantity $\nabla_{a} u_{b}$ will diverge.

The existence of such singular evolutions for $u_{a}$ signals a breakdown of the effective theory we are using. Perhaps it would be cured by including the term $\left(\nabla_{(a} u_{b)}\right)\left(\nabla^{(a} u^{b)}\right)$ in the action. (Without this term the action is insensitive to gradients for which the antisymmetrized derivative $\nabla_{[a} u_{b]}$ vanishes.) For the purposes of the present paper we shall not pursue this question, but it should be addressed.

\section{Cosmological solutions}

If $u^{a}$ shares the symmetry of a homogeneous isotropic cosmological metric, then $F_{a b}=0$. The presence of the aether field therefore has no influence on the cosmological evolution unless there are additional terms in the action beyond the minimal model. In Ref. [20] we examine some cosmological effects of coupling to a scalar field though a fourth spatial derivative term as discussed in Sect. VIB.

\section{Black hole solutions}

For a spherically symmetric black hole, a suitable congruence of geodesics is given by the radial free-fall trajectories that all have the same Killing energy, i.e. the same asymptotic velocity at spatial infinity. The same construction can even be applied in the case of a Kerr black hole, at least for the geodesics that are at rest at spatial infinity. This follows from the work of Ref. [21], in which this congruence is employed to construct a coordinate system for the Kerr metric using the time function $\tau$ mentioned above. 


\section{Spherically symmetric, static solutions}

Here we seek to characterize the general spherically symmetric, static solution. We shall find that, besides the mass, the metric in these solutions has an additional free parameter, the "aether charge".

Some of the linearized static, spherically symmetric solutions were previously studied in Ref. [13. Those authors examined the case where $u^{a}$ is spacelike, and while in four spacetime dimensions they restricted attention to vanishing Lagrange multiplier $\lambda$ and vanishing field strength $F_{a b}$. In the present work we treat the nonlinear case, considering only timelike $u^{a}$ and imposing no further restrictions on the fields.

Coordinates can be chosen so the line element takes the form

$$
d s^{2}=g_{t t} d t^{2}+g_{r r} d r^{2}-r^{2}\left(d \theta^{2}+\sin ^{2} \theta d \varphi^{2}\right),
$$

and the aether field takes the form

$$
u=u_{t}(r) d t+u_{r}(r) d r .
$$

The only potentially nonzero component of $F_{a b}$ is then $F_{r t}=\partial_{r} u_{t}$, and the constraint (3.9) implies

$$
g^{t t} u_{t}^{2}+g^{r r} u_{r}^{2}=1 .
$$

The aether field equation (3.8) in coordinate form reads

$$
\frac{1}{\sqrt{-g}} \partial_{\alpha}\left(\sqrt{-g} g^{\alpha \mu} g^{\beta \nu} F_{\mu \nu}\right)=-\frac{\lambda}{2 b_{1}} u^{\beta},
$$

or, taking into account the form of the metric (4.1),

$$
\frac{1}{\sqrt{-g}} \partial_{r}\left(\sqrt{-g} g^{r r} g^{\beta t} F_{r t}\right)=-\frac{\lambda}{b_{1}} u^{\beta} .
$$

The left hand side vanishes when $\beta=r$, hence the field equation implies that $\lambda u_{r}=0$, which in turn implies that either $u_{r}=0$ or $\lambda=0$. In the former case, $u^{a}$ is proportional to the timelike Killing field itself. There are thus two cases to consider.

If $\lambda \neq 0$ there are in fact no static solutions, unless the coefficients in the action are such that the charge to mass ratio of the dust is extremal. Recall that any solution to our theory is a solution to the charged dust theory. However under the influence of gravitational and electric forces, the non-extremal charged dust cannot remain static, since there is no pressure 22.23.

If $\lambda=0$ then (cf. Sect. IV A) these are just the spherically symmetric static Einstein-Maxwell solutions, i.e. the Reissner-Nordstrom solutions, in a spherically symmetric, static gauge with fixed norm (3.11). Such a gauge always exists, at least locally. Consider the gauge transform $A_{\mu}=A_{\mu}^{\prime}+\alpha_{, \mu}$ of an arbitrary vector potential $A_{\mu}^{\prime}$. To maintain spherical symmetry and time independence we must have $\alpha=\beta t+\gamma(r)$ (using the coordinates in
(4.1)), so that $A_{t}=A_{t}^{\prime}+\beta$ and $A_{r}=A_{r}^{\prime}+\gamma_{, r}$. The normalization (3.11) then implies

$$
\gamma_{, r}=-A_{r}^{\prime} \pm \sqrt{g^{t t}\left[g^{t t}\left(A_{t}^{\prime}+\beta\right)^{2}-4 b_{1}\right]},
$$

where we have used the fact that for the ReissnerNordstrom metrics $g_{r r}=-1 / g_{t t}$. In any region where $g^{t t}$ and $A_{t}$ are bounded one can always choose $\beta$ large enough so that the radical is real, and then $\gamma(r)$ can be found by integration.

If a horizon is present $g^{t t}$ diverges and it is not clear from the preceding discussion whether the unit timelike gauge can be accessed in a smooth way across the horizon. Indeed it can, however the maximal extension of the region over which such a gauge can be accessed depends on the parameter $b_{1}$. This can also be understood from the equivalence with a charged dust solution. The radial congruence $u^{a}$ must satisfy the Lorentz force equation (3.16), and this congruence can be nonsingular and time-independent only if the trajectories are monotonic in the $r$ coordinate. In general, however, the trajectories bounce inside the black hole.

\section{Comparison with observation}

To compare with observation it would be necessary to determine which of the above solutions to use in the presence of a spherically symmetric static source such as a planet, star, or black hole. The metric associated with one of these objects depends on its "aether charge" the charge of the Reissner-Nordstrom solution - which is determined by the "charge" of the "aether dust" that fell in when the object condensed. The choice is determined by the initial conditions on $\lambda$, which are presumably cosmological in origin. We have no theory of these initial conditions at this stage, but agreement with observations can put a bound on the amount of aether charge. If this charge is zero, then we have the usual Schwarzschild solution of general relativity (and, as discussed in section IV B, the aether field is the tangent field to a hypersurface orthogonal congruence of timelike geodesics), which of course agrees with observations.

\section{LINEARIZED THEORY}

In this section we study the linearized equations defined by expanding about a background solution,

$$
\begin{aligned}
g_{a b} & =g_{a b}^{(0)}+h_{a b} \\
u_{a} & =u_{a}^{(0)}+v_{a}, \\
\lambda & =\lambda^{(0)}+\lambda^{(1)} .
\end{aligned}
$$

For the background we take the flat metric $g_{a b}^{(0)}=\eta_{a b}$ and a constant $u_{a}^{(0)}$. In this background solution the 
equations of motion imply that the Lagrange multiplier $\lambda^{(0)}$ must vanish, hence we shall use the letter $\lambda$ for the perturbation $\lambda^{(1)}$. In this section we use the flat background metric to raise and lower indices. Note that we use the perturbation of the covariant vector $u_{a}$ to define the perturbation $v_{a}$.

We choose cartesian coordinates $\left(x^{0}, x^{i}\right), i=1,2,3$, in which the components of $\eta_{a b}$ are $\operatorname{diag}(1,-1,-1,-1)$ and those of $u_{a}^{(0)}$ are $(1,0,0,0)$. The linearized field equations for this theory were first written down in a general gauge in Ref. 13], who also pointed out that the Lorentz gauge can be accessed using the linearized diffeomorphism invariance of the action. The Lorentz gauge condition for the metric perturbation is $\bar{h}_{a b}{ }^{, b}=0$, where $\bar{h}_{a b} \equiv h_{a b}-\frac{1}{2} h \eta_{a b}$ is the trace-reversed metric perturbation. In this gauge, the linearized equations of motion are

$$
\begin{gathered}
\square \bar{h}_{a b}=2 \frac{\lambda}{a_{1}} u_{a}^{(0)} u_{b}^{(0)} \\
\square v_{b}-\partial_{b}\left(\partial^{a} v_{a}\right)=-\frac{\lambda}{2 b_{1}} u_{b}^{(0)} \\
-h_{a b} u^{(0) a} u^{(0) b}+2 u^{(0) b} v_{b}=0
\end{gathered}
$$

In a source-free region, the residual gauge freedom is usually employed to set $\bar{h}_{0 i}=h_{0 i}=0$ and $\bar{h}=-h=0$. 24. The possibility of doing so depends on the fact that these quantities satisfy the wave equation in general relativity. In our case, the components $h_{0 i}$ satisfy the wave equation, however $h$ does not, due to the source term on the right hand side of (5.4) that is always present (even outside matter) unless $\lambda=0$. This source corresponds to the energy density of the "charged dust", and we wish to allow for the presence of this term. Therefore, rather than setting $h$ to zero, we choose to set to zero the trace of the spatial part, $\bar{h}_{i}^{i}$, which does satisfy the wave equation. The proof that this can be done follows the same logic as in the usual case. This gauge condition implies $\bar{h}=\bar{h}_{00}$, hence $h_{00}=\bar{h}_{00}-\frac{1}{2} \bar{h} \eta_{00}=\frac{1}{2} \bar{h}_{00}$, and the Lorentz gauge condition implies $\partial_{0} \bar{h}_{00}=0$ and $\partial_{i} \bar{h}_{i j}=0$. Thus $h_{00}$ is time-independent and the spatial part $\bar{h}_{i j}$ is a transverse traceless solution to the wave equation. This is not quite the same as the usual transverse traceless gauge in general relativity however, since $h_{i j}=\bar{h}_{i j}-h_{00} \delta_{i j}$, so $h_{i j}$ is not transverse unless $\partial_{i} h_{00}=0$, and $h=-2 h_{00} \neq 0$.

It remains to consider the linearized equations for $h_{00}$, $v_{a}$, and $\lambda$. The 00-component of the metric equation (5.4) and the constraint equation (5.6) determine $\lambda$ and $v_{0}$ in terms of $h_{00}$ :

$$
\begin{aligned}
\lambda & =-a_{1} \nabla^{2} h_{00} \\
v_{0} & =-\frac{1}{2} h_{00} .
\end{aligned}
$$

Using the time-independence of $v_{0}$, the time and space components of the aether equation (5.5) read

$$
\begin{aligned}
-\nabla^{2} v_{0}-\partial_{0}\left(\partial^{i} v_{i}\right) & =-\frac{\lambda}{2 b_{1}} \\
\square v_{i}-\partial_{i}\left(\partial^{j} v_{j}\right) & =0 .
\end{aligned}
$$

Let us decompose $v_{i}$ into transverse and longitudinal parts, $v_{i}=v_{i}^{T}+v_{i}^{L}$, where $\partial^{i} v_{i}^{T}=0$ and $v_{i}^{L}=\partial_{i} f$ for some scalar field $f$. Then (5.10) implies that the transverse part satisfies the wave equation, $\square v_{i}^{T}=0$, so the the aether field has two transverse massless modes.

As for the longitudinal part $v_{i}^{L}$, equation (5.9) implies

$$
\partial_{0}\left(\partial^{i} v_{i}^{L}\right)=\frac{b_{1}-a_{1}}{2 b_{1}} \nabla^{2} h_{00}
$$

so in particular $v_{i}^{L}$ has at most linear time dependence. (The same conclusion follows from the divergence of Eq. (5.10).) Thus $v_{i}^{L}=\partial_{i} a(x) t+\partial_{i} b(x)$, where $a(x)$ is determined by $h_{00}$ and $b(x)$ is arbitrary.

In summary, the perturbation spectrum consists of two massless transverse traceless modes of $\bar{h}_{i j}$, and two massless transverse modes of $v_{i}$. In addition there is a mode in which $h_{00}$ is an arbitrarily specified time-independent function, which determines $v_{0}, \lambda$, and the time derivative of the longitudinal part $v_{i}^{L}$. The time-independent part of $v_{i}^{L}$ is also arbitrary. This last freedom corresponds to the linearization of the restricted gauge symmetry (3.5).

The longitudinal mode looks very strange at first sight. In the charged dust interpretation, the dust energy density is adjusted to produce an arbitrary gravitational potential $h_{00}$, and the perturbed metric, electromagnetic field, and charge density are all time independent, while the perturbed dust world lines are time dependent. This is a peculiarity of the first order perturbative solution however. No exact solution shares this property, as can be easily seen from the aether field equation (3.8). If the left hand side is invariant with respect to a timelike Killing field, and if $\lambda$ is also invariant, then so must be $u^{b}$. Evidently the higher order terms in the equations of motion induce time dependence into the solution. A similar phenomenon can be seen upon expanding the simpler Einstein-neutral dust system about the flat space solution with constant dust 4-velocity and vanishing density. As in our case, the dust density perturbation can set up any static metric perturbation, and the linearized geodesic equation for the dust yields a time-dependent dust velocity perturbation.

\section{MATTER COUPLINGS}

We have so far considered only the terms in the action involving the metric and the aether field and up to two derivatives. Suppose a matter term $S_{\text {mat }}\left[g_{a b}, u^{a}, \psi\right]$ is added to the action $S_{\min }\left[g_{a b}, u^{a}, \lambda\right]$ of the minimal theory (3.3), where $\psi$ stands for a generic matter field. The variation of $S_{m a t}$ with respect to the metric produces an additional contribution to the stress-energy tensor, and the variation with respect to $u^{a}$ produces an additional 
term in the current on the right hand side of (3.8). The resulting field equation takes the form

$$
\nabla^{a} F_{a b}=-\frac{1}{2 b_{1}}\left(\lambda u_{b}+\frac{1}{2} \frac{\delta S_{m a t}}{\delta u^{b}}\right) .
$$

The identity $\nabla^{a} \nabla^{b} F_{a b}=0$ then implies

$$
u^{a} \nabla_{a} \lambda=-\lambda \nabla_{a} u^{a}-\frac{1}{2} \nabla^{a} \frac{\delta S_{m a t}}{\delta u^{a}},
$$

which shows that now even if $\lambda$ is initially zero it need not remain zero. In the presence of such matter couplings the equivalence to charged dust is lost.

We now consider specific types of matter couplings, first of dimension less than or equal to four, and next of dimension greater than four.

\section{A. Couplings of dimension $\leq 4$}

A complete classification of Lorentz violating, gaugeinvariant extensions of the $\mathrm{SU}(3) \times \mathrm{SU}(2) \times \mathrm{U}(1)$ minimal standard model has been given by Colladay and Kostelecký [25], restricting attention to operators whose mass dimension is less than or equal to four, so as to preserve power-counting renormalizability. This class of low energy effective actions includes both $C P T$-even and $C P T$ odd terms, and involves various coupling tensors with "generation" indices allowing for mixing of fermions from different generations. These coupling tensors are supposed to be Lorentz violating vacuum expectation values arising in a theory with a fundamental underlying Lorentz symmetry.

Here we consider the above class of Lorentz violating terms, keeping only those couplings that can be constructed with the aether field $u^{a}$. With this restriction the antisymmetric tensor couplings are excluded, which rules out Lorentz-violating Yukawa couplings and couplings of gauge field strengths to Higgs bilinears, and limits the form of modifications of the gauge field kinetic terms. Invariance under time reversal $u^{a} \rightarrow-u^{a}$ would be required if the physical significance of the aether is only to define a preferred frame and not a preferred local time orientation. If we accordingly further assume this symmetry, all the CPT-odd terms are excluded, which rules out terms with a vector coupled to fermion or Higgs currents, gauge field Chern-Simons currents, and the $\mathrm{U}(1)$ potential.

The only possibilities remaining after all these restrictions have been imposed are the modifications of the fermion, gauge field, and Higgs kinetic terms:

$$
\begin{gathered}
\frac{1}{2} i\left(c_{L}\right)_{I J} u^{a} u^{b} \bar{L}_{I} \gamma_{a} D_{b} L_{J}+\text { h.c. }+\ldots \\
-\frac{1}{4} c_{B} u^{a} u^{b} g^{m n} B_{a m} B_{b n}+\ldots \\
\frac{1}{2} c_{\Phi} u^{a} u^{b}\left(D_{a} \Phi\right)^{\dagger} D_{b} \Phi .
\end{gathered}
$$

The indices $I, J$ in (6.3) are generational indices, and the coupling constants $\left(c_{L}\right)_{I J}, c_{B}$, and $c_{\Phi}$ are all dimensionless. The ellipses in (6.3) stand for similar terms for the other fermions, while those in (6.4) stand for similar terms for the other gauge fields.

Such additional kinetic terms modify the propagation speed of the various fields. For example, the propagation speed for the Higgs field, with respect to the preferred frame, is $\left(1+c_{\Phi}\right)^{-1 / 2}$, which is less or more than the speed of light if $c_{\Phi}$ is positive or negative respectively. The coupling constants must therefore be small numbers for fields whose propagator has been measured accurately. It would be interesting to determine what limits can be placed on these coefficients, particularly for fields such as the Higgs or gluons whose propagators are presumably not yet so well measured.

\section{B. Couplings of dimension $>4$}

Once the restriction to terms of dimension 4 or less is dropped, the possibilities for Lorentz violating terms like those for Lorentz invariant ones - are endless. Here we would like to consider just two types, which illustrate different possibilities that arise in the presence of Lorentz symmetry breaking.

\section{Modified kinetic terms}

If the coupling coefficient for a Lorentz violating kinetic term like (6.3.6.5) is field dependent and polynomial, rather than a constant, then the term is a dimension $>4$ operator. In this case it is possible that the coefficient was larger in the early universe than it is today, due to the cosmological evolution of the field(s) on which the coupling function depends. This provides an alternate approach to constructing generally covariant, variable speed of light cosmologies. Approaches using a vector [5] or a scalar [6,7] to define the preferred frame have been the subject of some recent papers.

\section{Modified dispersion}

Next we consider a deviation from Lorentz invariance that becomes strong only at high wavevectors. In the early universe, when the fields were highly excited at large wavevectors, the gravitational effects of such a deviation could have been of paramount importance. The study of a model incorporating such effects is left to another paper [20]. Here we indicate only an example of a term in the Lagrangian that produces high frequency dispersion in the propagation of a matter field, and we display the the form of the resulting contribution to the energy-momentum tensor. 
Consequences of non-Lorentz invariant high frequency dispersion for the Hawking effect have previously been studied using $1+1$ dimensional model field theories in which higher spatial derivative terms are added to the action (for a review see [14]), and recently such models have been generalized to field theory in the background of a $3+1$ dimensional Robertson-Walker spacetime in order to study the consequences for the spectrum of primordial density fluctuations in inflationary cosmology [26. These models can be extended to an arbitrary $3+1$ dimensional setting, preserving general covariance as well as spatial rotation symmetry in the local preferred frame. As an example consider the Lagrangian

$$
\mathcal{L}_{\varphi}=\frac{1}{2}\left(\nabla^{a} \varphi \nabla_{a} \varphi+k_{0}^{-2}\left(D^{2} \varphi\right)^{2}\right)
$$

Here $k_{0}$ is a constant with the dimensions of inverse length which sets the scale for deviations from Lorentz invariance, and $D^{2}$ is the covariant spatial Laplacian, i.e.,

$$
D^{2} \varphi=-D^{a} D_{a} \varphi=-q^{a c} \nabla_{a}\left(q_{c}^{b} \nabla_{b} \varphi\right)
$$

where $D_{a}$ is the spatial covariant derivative operator 24] and $q_{a b}$ is the spatial metric (3.6).

The $u^{a}$-dependence of the Lagrangian (6.6) produces a "matter" term in the aether field equation (6.1). The energy-momentum tensor for this Lagrangian is

$$
\begin{aligned}
& T_{a b}=\nabla_{a} \varphi \nabla_{b} \varphi-\mathcal{L}_{\varphi} g_{a b} \\
& -k_{0}^{-2}\left[2 D^{2} \varphi u^{m} u_{(a} \nabla_{|m|} D_{b)} \varphi+2 \nabla_{m}\left(D^{2} \varphi q_{(a}{ }^{m}\right) \nabla_{b)} \varphi\right. \\
& \left.\quad-\nabla^{m}\left(q_{a b} D^{2} \varphi D_{m} \varphi\right)\right] .
\end{aligned}
$$

In Ref. 20] we evaluate the expectation value of this energy-momentum tensor in a thermal state in flat spacetime, which allows us to determine the modification of the equation of state produced by the fourth derivative term. This equation of state is then be used to study how the cosmological evolution is affected by the high frequency dispersion.

\section{DISCUSSION}

We have made an initial attempt to study the possible consequences of incorporating a preferred frame - the aether-into a generally covariant theory. With the action adopted in this paper the aether vector generically develops gradient singularities even when the metric is perfectly regular. We take this as a sign that the theory is unphysical as an effective theory (although if the aether sector is ignored the theory can be made to agree with observations with an appropriate choice of initial conditions, i.e. by setting $F_{a b}$ to zero.) The primary open questions are $(i)$ what determines the initial values of the aether field and the Lagrange multiplier field, and (ii) are the gradient singularities, which appear to be generic in the evolution of the aether, eliminated by including a symmetrized derivative term $\left(\nabla_{(a} u_{b)}\right)\left(\nabla^{(a} u^{b)}\right)$ in the action along with the antisymmetrized derivative term used in this paper? It is plausible that adding the symmetrized derivative term will have a significant effect, since with it the action is sensitive to the existence of any large gradients.

\section{ACKNOWLEDGEMENTS}

We are grateful to V.A. Kostelecký, M. Luty, and R.P. Woodard for helpful discussions. This work was supported in part by the National Science Foundation under grant No. PHY98-00967.

[1] R.P. Woodard, "A Canonical formalism for Lagrangians with nonlocality of finite extent", Phys. Rev. A62, 052105 (2000); see also J. Llosa and J. Vives, "Hamiltonian formalism for nonlocal Lagrangians," J. Math. Phys. 35, 2856 (1994).

[2] C.M. Will and K. Nordvedt, Jr., "Conservation laws and preferred frames in relativistic gravity. I. Preferred frame theories and an extended PPN formalism, Astrophys. J. 177, 757 (1972).

[3] K. Nordvedt, Jr. and C.M. Will, "Conservation laws and preferred frames in relativistic gravity. II. Experimental evidence to rule out preferred frame theories of gravity", Astrophys. J. 177, 775 (1972).

[4] R.W. Hellings and K. Nordvedt, Jr., "Vector-metric theory of gravity", Phys. Rev. D7, 3593 (1973).

[5] M.A. Clayton and J.W. Moffat, "Dynamical mechanism for varying light velocity as a solution to cosmological problems," Phys. Lett. B460, 263 (1999).

[6] M.A. Clayton and J.W. Moffat, "Scalar-tensor gravity theory for dynamical light velocity," Phys. Lett. B477, 269 (2000).

[7] B.A. Bassett, S. Liberati, C. Molina-Paris and M. Visser, "Geometrodynamics of variable-speed-of-light cosmologies," Phys. Rev. D 62, 103518 (2000).

[8] D. Finkelstein, J.M. Jauch, S. Schiminovich and D. Speiser, "Principle of general Q-covariance", J. Math. Phys. 4, 788 (1963).

[9] T. Appelquist and C. Bernard, "Strongly interacting Higgs bosons," Phys. Rev. D22, 200 (1980).

[10] J.C. Dell and T. Jacobson, unpublished.

[11] P.A.M. Dirac, "A New classical theory of electrons" (I,II,III), Proc. Roy. Soc. A 209, 291 (1951); 212, 330 (1951); 223, 438 (1954).

[12] See, for example, M. Gasperini, "Singularity prevention and broken Lorentz symmetry", Class. Quantum Grav. 4, 485 (1987); "Repulsive gravity in the very early Universe", Gen. Rel. Grav. 30, 1703 (1998); and references therein. 
[13] V.A. Kostelecký and S. Samuel, "Gravitational phenomenology in higher dimensional theories and strings," Phys. Rev. D40, 1886 (1989).

[14] T. Jacobson, "Trans-Planckian redshifts and the substance of the space-time river," Prog. Theor. Phys. Supp. 136, 1 (1999).

[15] J.D. Barrow and F.J. Tipler, "Analysis of the generic singularity studies by Belinskii, Khalatnikov, and Lifschitz", Phys. Rep. 56371 (1979).

[16] R.M. Wald and P. Yip, "On the existence of simultaneous synchronous coordinates in spacetimes with spacelike singularities", J. Math. Phys. 22, 2659 (1981).

[17] L. Andersson, G.J. Galloway and R. Howard, "The Cosmological Time Function," Class. Quant. Grav. 15, 309 (1998).

[18] C.M. Will, Theory and Experiment in Gravitational Physics, (Cambridge Univ. Press, Cambridge, 1993).

[19] We thank J.C. Dell for pointing out our earlier omission of this term.

[20] T. Jacobson and D. Mattingly, "Generally covariant model of a scalar field with high frequency dispersionand the cosmological horizon problem," Phys. Rev. D 63, 041502 (2001).

[21] C. Doran, "A new form of the Kerr solution," Phys. Rev. D61, 067503 (2000).

[22] W.B. Bonner, "Equilibrium of a charged sphere", Mon. Not. Roy. Astr. Soc. 129, 443 (1965).

[23] U.K. De and A.K. Raychaudhuri, "Static distribution of charged dust in general relativity", Proc. Roy. Soc. A 303, 97 (1968).

[24] R.M. Wald, General Relativity, (Univ. Chicago Press, Chicago, 1984).

[25] D. Colladay and V. A. Kostelecky, "Lorentz-violating extension of the standard model," Phys. Rev. D58, 116002 (1998).

[26] J. Martin and R. H. Brandenberger, "The transPlanckian problem of inflationary cosmology," hepth/0005209; J. C. Niemeyer, "Inflation with a Planckscale frequency cutoff," astro-ph/0005533; T. Tanaka, "A comment on trans-Planckian physics in inflationary universe," astro-ph/0012431; L. Mersini, M. BasteroGil and P. Kanti, "Relic dark energy from transPlanckian regime," hep-ph/0101210; J. C. Niemeyer and R. Parentani, "Trans-Planckian dispersion and scale-invariance of inflationary perturbations," astroph/0101451; A. A. Starobinsky, "Robustness of the inflationary perturbation spectrum to trans-Planckian physics," Pisma Zh. Eksp. Teor. Fiz. 73, 415 (2001) astro-ph/0104043; R. Easther, B. R. Greene, W. H. Kinney and G. Shiu, "Inflation as a probe of short distance physics," hep-th/0104102. 